\title{
Article \\ Clinical Association between Gout and Parkinson's Disease: A Nationwide Population-Based Cohort Study in Korea
}

\author{
Ji Hyoun Kim ${ }^{1}$, In Ah Choi ${ }^{1,2}$, Aryun Kim ${ }^{3} \mathbb{C}$ and Gilwon Kang ${ }^{4, *}$ \\ 1 Division of Rheumatology, Department of Internal Medicine, Chungbuk National University Hospital, \\ Cheongju 28644, Korea; jeehyounee@cbnuh.or.kr (J.H.K.); iachoi@cbnu.ac.kr (I.A.C.) \\ 2 Department of Internal Medicine, College of Medicine, Chungbuk National University, \\ Cheongju 28644, Korea \\ 3 Department of Neurology, Chungbuk National University Hospital, Cheongju 28644, Korea; \\ mypioneer97@gmail.com \\ 4 Department of Health Information and Management, College of Medicine, Chungbuk National University, \\ Cheongju 28644, Korea \\ * Correspondence: gilwon67@chungbuk.ac.kr
}

\section{check for} updates

Citation: Kim, J.H.; Choi, I.A.; Kim, A.; Kang, G. Clinical Association between Gout and Parkinson's Disease: A Nationwide Population-Based Cohort Study in Korea. Medicina 2021, 57, 1292. https://doi.org/10.3390/ medicina57121292

Academic Editors: Wisit Cheungpasitporn, Charat Thongprayoon, Wisit Kaewput and Pattharawin Pattharanitima

Received: 14 October 2021

Accepted: 22 November 2021

Published: 24 November 2021

Publisher's Note: MDPI stays neutral with regard to jurisdictional claims in published maps and institutional affiliations.

Copyright: (c) 2021 by the authors. Licensee MDPI, Basel, Switzerland. This article is an open access article distributed under the terms and conditions of the Creative Commons Attribution (CC BY) license (https:// creativecommons.org/licenses/by/ $4.0 /)$.

\begin{abstract}
Background and Objectives: This retrospective cohort study aimed to investigate the association between gout and Parkinson's disease (PD) in Korea. Materials and Methods: Overall, 327,160 patients with gout and 327,160 age- and sex-matched controls were selected from the Korean National Health Insurance Service (NHIS) database. PD incidence was evaluated by reviewing NHIS records during the period from 2002 to 2019. Patients with a diagnosis of gout (International Classification of Diseases-10 (ICD-10), M10) who were prescribed medications for gout, including colchicine, allopurinol, febuxostat, and benzbromarone for at least 90 days were selected. Patients with PD who were assigned a diagnosis code (ICD-G20) and were registered in the rare incurable diseases (RID) system were extracted. Results: During follow-up, 912 patients with gout and 929 control participants developed PD. The incidence rate (IR) of overall PD (per 1000 person-years) was not significantly different between both groups ( 0.35 vs. 0.36 in gout and control groups, respectively). The incidence rate ratio (IRR) was 0.98 (95\% CI: 0.89-1.07). The cumulative incidence of PD was not significantly different between the groups. No association between gout and PD was identified in univariate analysis ( $\mathrm{HR}=1.00,95 \% \mathrm{CI}: 0.91-1.10, p=0.935)$. HR increased significantly with old age ( $\mathrm{HR}=92.08,198$, and 235.2 for $60-69$ years, 70-79 years, and over 80 years, respectively), female sex $(\mathrm{HR}=1.21,95 \% \mathrm{CI}: 1.07-1.37, p=0.002)$, stroke $(\mathrm{HR}=1.95,95 \% \mathrm{CI}: 1.76-2.16, p<0.001)$, and hypertension ( $\mathrm{HR}=1.16,95 \% \mathrm{CI}: 1.01-1.34, p=0.04)$. Dyslipidemia exhibited an inverse result for PD (HR $=0.6,95 \%$ CI: $0.52-0.68, p<0.001)$. Conclusions: This population-based study did not identify an association between gout and PD. Age, female sex, stroke, and hypertension were identified as independent risk factors for PD, and dyslipidemia demonstrated an inverse result for PD.
\end{abstract}

Keywords: gout; uric acid; Parkinson's disease

\section{Introduction}

Parkinson's disease (PD) is a chronic neurodegenerative disease caused by prominent dopaminergic neuron loss and the accumulation of $\alpha$-synuclein-containing Lewy bodies and Lewy neurites [1]. The characteristic clinical symptoms of PD are motor symptoms, including tremors, rigidity, and bradykinesia [2]. PD is the second most-common neurodegenerative disease, after Alzheimer's disease [3], and affects 1-2\% of individuals over 65 years of age [4], with a reported prevalence of 315 per 100,000 persons of all ages [4]. A previous study reported that the prevalence of PD was lower in Asian populations than in other ethnic groups [5]. Nevertheless, the prevalence of PD in Korea was reported to be 156.9 per 100,000 persons in 2012 and 181.3 per 100,000 persons in 2015 , highlighting the increase in PD diagnosis over the past 3 years [6]. 
Gout is a chronic inflammatory disease characterized by severe pain caused by joint and soft tissue inflammation [7]. Previous studies have reported that uric acid may possess antioxidant properties and that low serum uric acid levels are associated with an increased risk of several neurodegenerative diseases [8-10]. Further, hyperuricemia has been reported to reduce the risk of PD [11] and inflammatory arthritis, including gout, has been identified as a risk factor for PD. However, the relationship between gout and PD is still unclear. This study aimed to investigate the association between gout and PD [12-14] by using large-scale data.

\section{Materials and Methods}

\subsection{Database and Study Population}

This retrospective cohort study used data from the Korean National Health Insurance Service (NHIS) database. The NHIS was launched in 2000 as the single insurer covering the entire population of Korea by integrating 375 insurance associations [15]. The NHIS comprises the eligibility database, national health screening database, basic demographic variables (i.e., age, sex, residential area, type of health insurance, etc.), International Classification of Diseases-10 (ICD-10) disease codes, health care utilization (diagnosis, length of stay, treatment costs, and services received), and prescription records (drug code, days prescribed, and daily dosage) [16].

\subsection{Study Cohort Selection and Parkinson's Disease Assessment}

Data from the NHIS database covering the period from 2002 to 2019 were used. Data from 2002 were excluded as a wash-out period for new patient extraction, and data from 2018 and 2019 were also excluded to secure a follow-up period of at least 2 years. In total, 628,565 participants with a diagnosis of gout (ICD-10, M10) who were prescribed medications for gout, such as colchicine, allopurinol, febuxostat, and benzbromarone for at least 90 days were selected from the NHIS database. In this study, in order to improve the reliability of the diagnosis of patients with gout, the gout diagnosis ICD code was matched with the gout-specific medication code. As this was a study that used diagnostic codes and medication prescription codes, laboratory findings, including uric acid levels, could not be confirmed.

Patients with PD who were assigned a diagnosis code (ICD-G20) and were registered in the rare incurable diseases (RID) system were extracted. Patients with secondary Parkinsonism (G21, G22) or dyskinesia due to other causes (G23-G25) were excluded. The Korean government provides financial support to patients with rare, incurable rheumatic diseases that are specially registered and have been managed since 2009. To be eligible for registration in the RID system, patients must meet the diagnostic criteria of each RID and are carefully reviewed by the corresponding healthcare institution. Only a neurologist or neurosurgeon can register patients with PD according to the United Kingdom PD Society Brain Bank criteria on the RID system. The diagnosis is also made by excluding other forms of secondary parkinsonism. Therefore, diagnoses of patients with PD registered in the RID system are considered highly reliable.

In this study, 215,711 patients with cancer, 85,331 patients with end-stage renal disease (ESRD), and 363 previous patients with PD were removed. A final total of 327,160 new gout patients were extracted. The control cohort was defined as patients who had never received a gout diagnostic code and had never been administered any gout medication. Age and sex (1:1) propensity score matching was performed in the selected control cohort. The flow chart for study cohort selection is presented in Figure 1. 


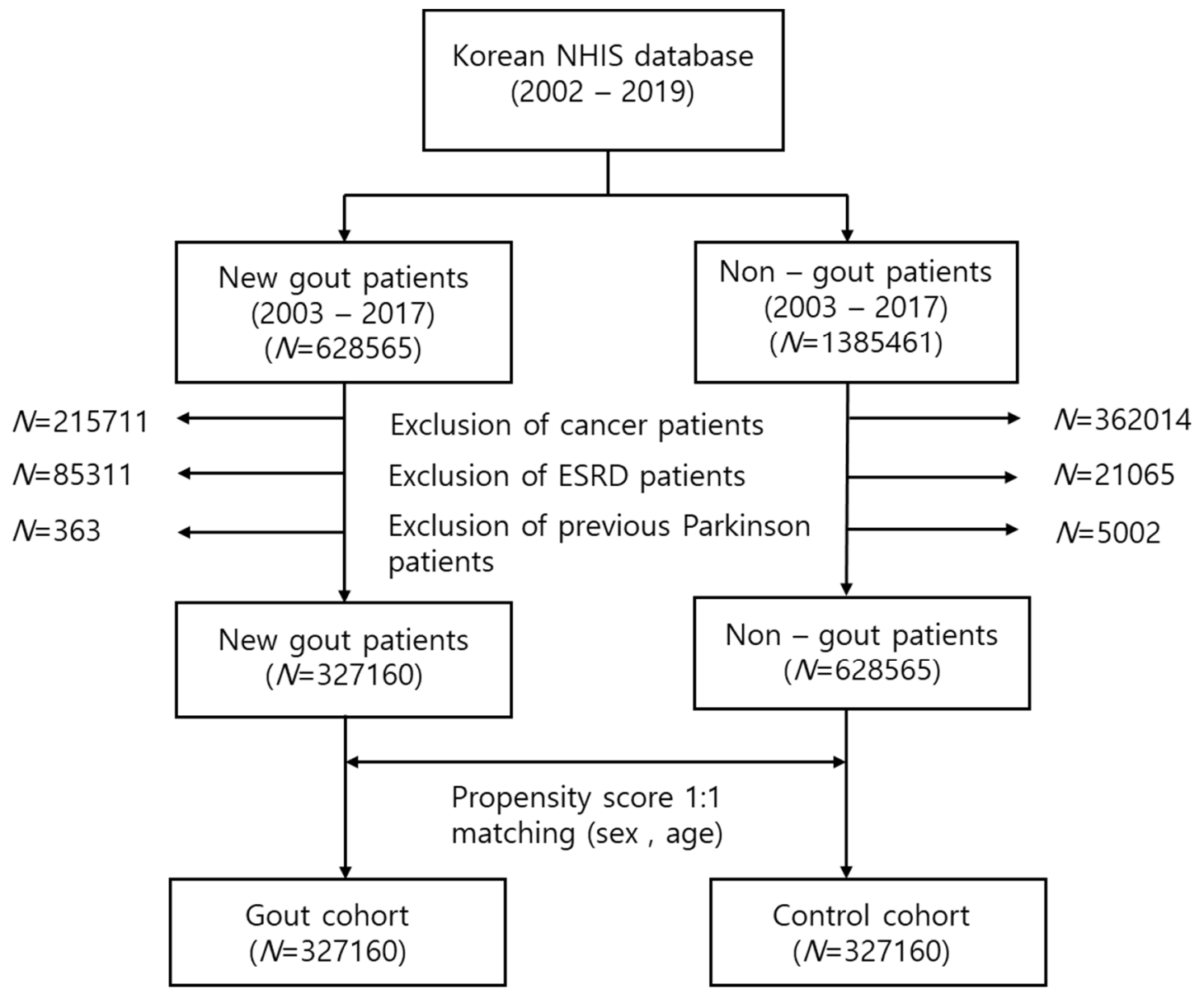

Figure 1. Flow chart for the selection of the study population from the National Health Insurance Service database. In total, 327,160 patients with gout and 327,160 control participants were compared via propensity score matching.

\subsection{Statistical Analysis}

The baseline demographics and clinical characteristics of the patients with gout and the controls at baseline were analyzed using a chi-square test or a Student's $t$-test. The Cox proportional hazards model was used to evaluate the effect of gout on the risk of PD, and the results are presented as hazard ratios (HRs) with $95 \%$ confidence intervals (CIs) using different adjustment models for potential confounders. There was no multicollinearity between variables, and the proportional hazard assumption was met. Cumulative PD incidence was determined using the Kaplan-Meier method and compared between the two cohorts using the log-rank test. Analyses were performed using the SAS statistical package version 9.4 (SAS Institute Inc., Cary, NC, USA) and R version 3.4.3 (R Foundation for Statistical Computing, Vienna, Austria). A $p$-value $<0.01$ was considered statistically significant.

\section{Results}

The database included medical information of almost all Korean individuals enrolled in the NHIS database. In total, 630,000 new patients diagnosed with gout were identified during the 18-year period from 2002 to 2019. A final total of 327,160 patients with gout satisfied the study criteria. Our analysis revealed the distribution of patients with gout in Korea according to age, with the following approximate numbers: $<30$ years $(\mathrm{n}=30,000)$, 30-39 years $(n=75,000), 40-49$ years $(n=85,000), 50-59$ years $(n=70,000), 60-69$ years $(\mathrm{n}=40,000), 70-79$ years $(\mathrm{n}=20,000)$, and $80-89$ years $(\mathrm{n}=6000)$. The age group with the highest prevalence was the 40-49 years age group. Most patients with gout were male $(304,162 ; 93 \%)$. No significant differences were observed in the mean age and sex 
distribution between the patients with gout and the controls, indicating that the groups were well matched. Participants with diabetes comprised $40 \%$ and $57 \%$ of the control and gout groups, respectively $(p<0.001)$. Hypertension $(40 \%$ in the control group vs. $65 \%$ in the gout group), dyslipidemia (62\% in the control group vs. $87 \%$ in the gout group), ischemic heart disease ( $22 \%$ in the control group vs. $31 \%$ in the gout group), and stroke ( $8.5 \%$ in the control group vs. $10.7 \%$ in the gout group) were significantly more common in patients with gout than in controls. The demographics and clinical characteristics of the study cohort at baseline are presented in Table 1 .

Table 1. Demographics and clinical characteristics of the study cohort.

\begin{tabular}{|c|c|c|c|}
\hline \multirow{2}{*}{ Variables } & Gout Patients & Controls & \multirow{2}{*}{$p$-Value } \\
\hline & $(N=327,160)$ & $(N=327,160)$ & \\
\hline \multicolumn{4}{|l|}{ Sex } \\
\hline Male & 304,162 & 304,162 & \\
\hline Female & 22,998 & 22,998 & \\
\hline \multicolumn{4}{|l|}{ Age (years) } \\
\hline$<30$ & 30,581 & 30,581 & \\
\hline $30-39$ & 75,265 & 75,265 & \\
\hline $40-49$ & 85,730 & 85,730 & \\
\hline $50-59$ & 70,912 & 70,912 & \\
\hline $60-69$ & 39,063 & 39,063 & \\
\hline $70-79$ & 19,638 & 19,638 & \\
\hline$\geq 80$ & 5.971 & 5.971 & \\
\hline \multicolumn{4}{|c|}{ Underlying diseases } \\
\hline Hypertension & $211,097(64.52 \%)$ & $140,890(43.06 \%)$ & $<0.001$ \\
\hline Diabetes & $186,198(56.91 \%)$ & $130,351(39.84 \%)$ & $<0.001$ \\
\hline Dyslipidemia & $284,794(87.05 \%)$ & $203,823(62.3 \%)$ & $<0.001$ \\
\hline $\begin{array}{l}\text { Ischemic heart } \\
\text { disease }\end{array}$ & $102,591(31.36 \%)$ & $73,162(22.36 \%)$ & $<0.001$ \\
\hline Stroke & $34,920(10.67 \%)$ & $27,777(8.49 \%)$ & $<0.001$ \\
\hline
\end{tabular}

Values are presented as number of patients (\%).

Table 2 presents the incidence of PD in patients with gout and in the control group. Overall, 912 patients with gout and 929 control participants developed PD. The incidence rate (IR) of overall PD (per 1000 person-years) was not significantly different between the two groups ( 0.35 in the gout group vs. 0.36 in the control group). The incidence rate ratio (IRR) was 0.98 (95\% CI: 0.89-1.07). A stratified analysis by sex and age did not reveal any significant differences between the groups (Table 2). In the analysis of the cumulative incidence of PD, no significant between-group differences were observed (Figure 2).

Table 2. Incidence of Parkinson's disease in patients with gout and in the controls, stratified by age group and sex.

\begin{tabular}{|c|c|c|c|c|c|c|c|}
\hline \multirow{2}{*}{ Variables } & \multicolumn{3}{|c|}{ Gout $(N=327,160)$} & \multicolumn{3}{|c|}{ Controls $(N=327,160)$} & \multirow{2}{*}{ IRR (95\% CI) } \\
\hline & $N, \%$ & PY & IR & $N, \%$ & PY & IR & \\
\hline Overall & 912 & 6544.07 & 0.35 & 929 & 6676.18 & 0.36 & $0.98(0.89-1.07)$ \\
\hline \multicolumn{8}{|l|}{ Sex } \\
\hline Male & 718 & 5437.98 & 0.29 & 748 & 5541.36 & 0.31 & $0.98(0.88-1.08)$ \\
\hline Female & 194 & 1106.09 & 1.32 & 181 & 1134.82 & 1.19 & $1.09(0.9-1.35)$ \\
\hline \multicolumn{8}{|l|}{ Age, years } \\
\hline $20-29$ & 2 & 8.18 & 0.01 & 3 & 11.45 & 0.01 & $0.67(0.11-4.0)$ \\
\hline $30-39$ & 14 & 87.16 & 0.02 & 17 & 139.21 & 0.03 & $0.82(0.41-1.67)$ \\
\hline $40-49$ & 60 & 441.84 & 0.08 & 66 & 524.01 & 0.09 & $0.91(0.64-1.28)$ \\
\hline $50-59$ & 164 & 1363.84 & 0.28 & 170 & 1334.36 & 0.3 & $0.95(0.77-1.18)$ \\
\hline $60-69$ & 325 & 2550.98 & 1.10 & 312 & 2475.13 & 1.07 & $1.03(0.88-1.2)$ \\
\hline $70-79$ & 277 & 1779.07 & 0.56 & 299 & 1952.53 & 2.41 & $0.91(0.78-1.08)$ \\
\hline$\geq 80$ & 70 & 313.01 & 2.53 & 62 & 239.48 & 2.40 & $1.05(0.75-1.48)$ \\
\hline
\end{tabular}




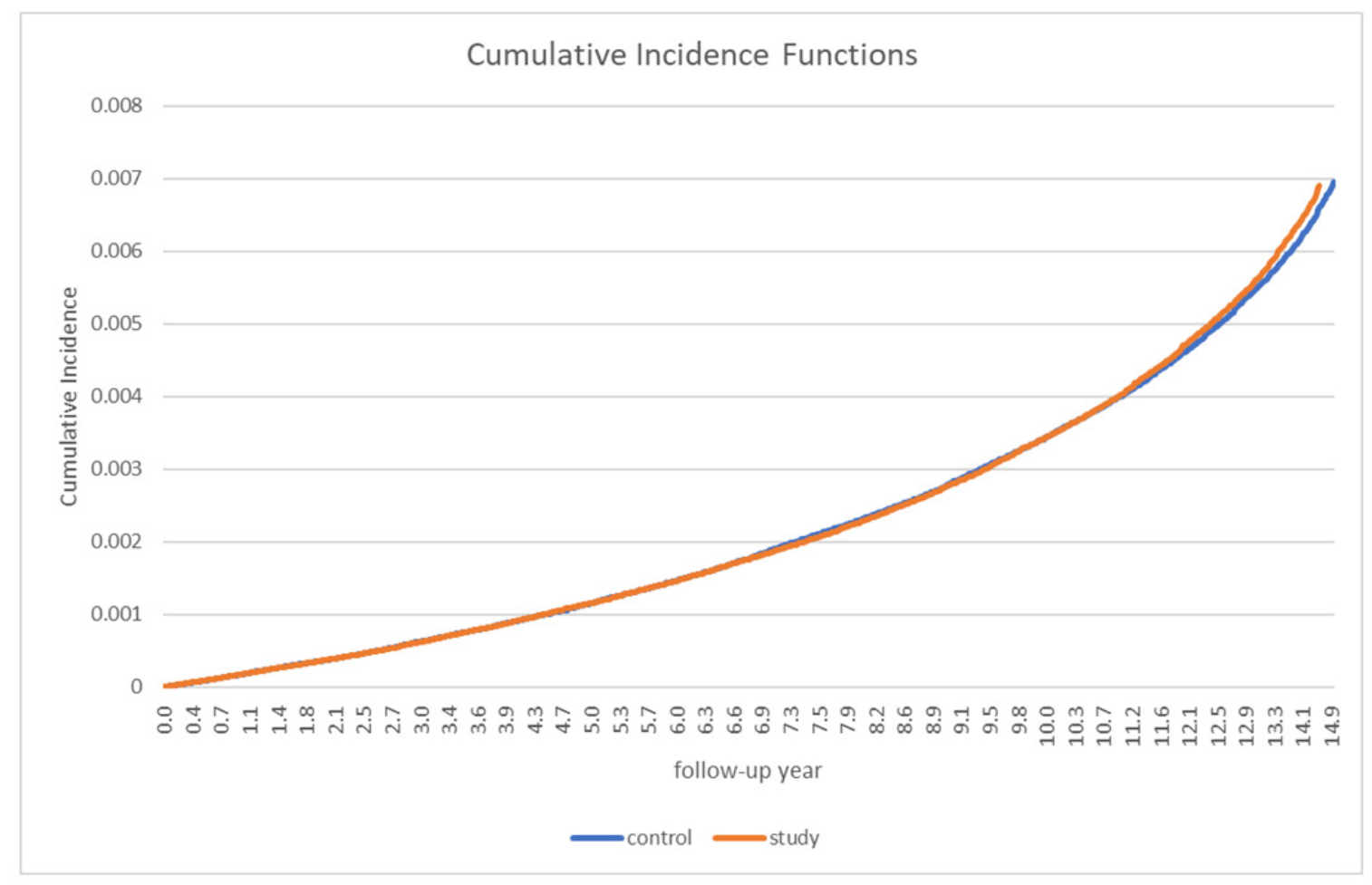

Figure 2. Cumulative Parkinson's disease incidence in patients with gout and the controls.

The association between gout and PD was not significant (HR = 1.00, 95\% CI: 0.91-1.10, $p=0.935$ ) (Table 3). Therefore, other potential risk factors for PD were investigated. The most significant result was the age of patients. We identified a significant association between the incidence of PD and aging $(\mathrm{HR}=92.08,198$, and 135.3 in 60-69 years, 70-79 years, and $>80$ years, respectively). Other factors that affected the incidence of PD were female sex $(\mathrm{HR}=1.21,95 \% \mathrm{CI}: 1.07-1.37, p=0.0024)$, stroke $(\mathrm{HR}=1.95,95 \%$ CI: 1.76-2.16, $p<0.001)$, and hypertension ( $\mathrm{HR}=1.16,95 \% \mathrm{CI}: 1.01-1.34, p=0.040)$. Notably, dyslipidemia exhibited inverse results for PD (HR $=0.6,95 \%$ CI: 0.52-0.68, $p<0.001)$ (Table 3).

Table 3. Cox proportional hazards model of risk factors for Parkinson's disease.

\begin{tabular}{lcccc}
\hline & Unadjusted HR (95\% CI) & $p$-Value & Adjusted HR (95\% CI) & $p$-Value \\
\hline Gout & $0.98(0.89-1.07)$ & 0.602 & $1.00(0.91-1.10)$ & 0.935 \\
Female sex & $4.731(4.22-5.30)$ & $<0.001$ & $1.21(1.07-1.37)$ & 0.002 \\
Age & & & & \\
$\quad 30-39$ & $2.29(0.89-5.9)$ & 0.085 & $2.33(0.91-6.00)$ & 0.079 \\
$40-49$ & $7.58(3.10-18.54)$ & $<0.001$ & $7.7(3.15-18.83)$ & $<0.001$ \\
$50-59$ & $27.63(43.35-251.96)$ & $<0.001$ & $26.95(11.12-65.34)$ & $<0.001$ \\
$60-69$ & $104.51(43.35-251.96)$ & $<0.001$ & $92.08(38.03-222.93)$ & $<0.001$ \\
$70-79$ & $256.07(106.17-617.61)$ & $<0.001$ & $197.5(81.39-479.21)$ & $<0.001$ \\
$\geq 80$ & $343.41(140.52-839.22)$ & $<0.001$ & $235.26(95.53-579.38)$ & $<0.001$ \\
Hypertension & $3.59(3.17-4.03)$ & $<0.001$ & $1.16(1.01-1.34)$ & 0.040 \\
Diabetes & $2.02(1.83-2.22)$ & $<0.001$ & $0.93(0.83-1.04)$ & 0.216 \\
Dyslipidemia & $1.25(1.11-1.40)$ & $<0.001$ & $0.6(0.52-0.68)$ & $<0.001$ \\
Ischemic heart disease & $2.37(2.17-2.60)$ & $<0.001$ & $0.99(0.90-1.1)$ & 0.998 \\
Stroke & $5.71(5.2-6.27)$ & $<0.001$ & $1.95(1.76-2.16)$ & $<0.001$ \\
\hline
\end{tabular}

HR: hazard ratio (using the Cox proportional hazards model), CI: confidence interval. 
We divided gout patients into male and female groups and performed a sub-analysis on comorbidities affecting PD. The risk was highest in stroke, HR 2.15 (95\% CI 1.91-2.41, $p<0.0001)$ in the male group and HR 1.39 (95\% CI 1.12-1.73, $p=0.0025)$ in the female group. In the female group, the risk of hypertension increased to HR 1.46 (95\% CI 0.96-2.22, $p=0.0793$ ), but there was no significance (Supplement Table S1).

\section{Discussion}

The purpose of this study was to determine the association between gout and the incidence of PD. We did not observe any significant differences in the incidence and risk of PD between the patients with gout and the controls using large-scale data. Previous studies have reported an association between hyperuricemia and PD. An analysis of US Medicare data of 1.7 million individuals from 2006 to 2012 revealed that hyperuricemia increased the risk of incidental PD ( $\mathrm{HR}=1.27,95 \%$ CI: 1.16-1.39) in individuals between 65 and 75 years of age [17]. In contrast, a retrospective study, using Taiwan's National Health Insurance Research Database, that analyzed 7900 patients with gout and 1:1-matched control participants without gout did not reveal any significant results $(\mathrm{HR}=1.01,95 \% \mathrm{CI}$ : 0.93-1.31) [18]. Similar to the Taiwanese study, we did not identify a significant association between gout and PD. However, $\mathrm{PD}$ is a relatively rare disease, with a lower prevalence in Asian populations than in other ethnic groups [5]. Therefore, these discrepant results may be due to genetic differences between Asians and other ethnic groups. Additionally, since our study only extracted patients with PD registered in the RID system, the patients included in this study predominantly comprised those who visited tertiary hospitals. Therefore, the incidence of PD may have been underestimated. In addition, in order to increase the reliability of the diagnosis for gout and to differentiate it from other studies, we defined gout groups as those who had a diagnosis code and continued gout medication for 3 months or more. Therefore, it can be expected that the patients with a relatively well-controlled uric acid level were also included in this study. It was inappropriate to evaluate the protective effect of uric acid on the development of PD. Further studies are needed to address this issue.

In our study, the incidence of cardiovascular comorbidities, including hypertension, dyslipidemia, ischemic heart disease, and stroke, were significantly increased among patients with gout compared to the control group. These results were consistent with previous studies [19-22].

In this study, we did not identify an association between gout and PD after adjusting for age, sex, and comorbidities. PD is a complex neurodegenerative disease and it can be affected by many other factors other than gout. Therefore, we investigated other potential factors affecting PD. The Cox proportional hazards model revealed that old age, female sex, stroke, and hypertension were independent risk factors for PD.

Han et al. [6] reported that the prevalence and incidence of PD were higher in women than in men. Other studies on Asian populations in Japan, China, and Taiwan revealed consistent results, with a reported female preponderance or no sex-related differences in the occurrence of PD [23-25]. In our study, the result identified female sex as a risk factor for PD, although only 7\% of the study's cohort was female. Conversely, studies in Europe and North/South America have reported opposing results, suggesting that sex differences may exist according to race/ethnicity [26-29].

We conducted a sub-analysis by sex group for risk factors affecting PD using the Cox proportional hazards model. The analysis result revealed that stroke was still an important risk factor, and that hypertension also tended to increase risk for PD in the female group. Therefore, we suggest that controlling hypertension, a modifiable risk factor, and preventing stroke can help lower the risk of PD, especially in females, who comprise the risk group.

Our study results revealed an inverse relationship between PD and dyslipidemia. The association between dyslipidemia and PD is controversial [30-34]. In this study, the HR of dyslipidemia for PD risk was $0.6(95 \%$ CI $0.52-0.68, p<0.001)$ in the whole of the patients, 
HR 0.6 in the male group $(95 \%$ CI $0.52-0.69, p<0.0001)$ and HR 0.59 in the female group (95\% CI 0.42-0.82, $p=0.0017)$. These results were consistent with two recent large cohort studies [32,33]. A possible explanation is as follows: cholesterol is involved in critical biological functions, including cellular repair, degeneration, and acts as a neurosteroid precursor. Potential beneficial roles of higher cholesterol levels in other neurodegenerative disorders have also been reported [35-39]. Nevertheless, the mechanisms of these effects have yet to be elucidated. Another potential mechanism involves the anti-inflammatory actions of statins, which reduce oxidative stress and neuro-inflammation [40,41].

A previous study reported several risk factors and protective factors for PD. For instance, cigarette smoking, coffee drinking, vitamin E intake, $\beta 2$-adrenoceptor agonist, and gout were suggested to exert protective effects [42]. Indeed, several reports have indicated that uric acid is a possible protective factor for PD [43,44]. However, the mechanisms underlying the inverse association between serum uric acid and PD remain unclear.

Uric acid is a product of purine metabolism and accounts for approximately $60 \%$ of free radical scavenging activity in human serum. Based on results from in vitro and in vivo experimental studies, uric acid may act as a neuroprotective agent by scavenging peroxynitrite-derived radicals, which are involved in the pathogenesis of neurodegenerative diseases $[43,44]$. Uric acid has also been reported to have iron-chelating properties. Iron is an essential element in cell metabolism and is involved in myelination and neurotransmission. The accumulation of iron in the substantia nigra, a region critically involved in PD, plays an important role in the death of dopaminergic neurons $[45,46]$. However, we did not examine this hypothesis in the current study.

Our study has several limitations. First, we were unable to collect data regarding participants' serum uric acid levels. Second, we did not investigate other environmental factors that could affect PD, such as residence, income, education level, drug use, smoking or drinking history, obesity, and other comorbidities. Furthermore, we included only patients who were administered gout-specific medications to increase the reliability of the diagnosis of gout. This is a limitation, and further studies on the effects of gout medications are needed. Nevertheless, this study represents the largest analysis of the current dataset so far. Further, the study results revealed the cumulative incidence of gout according to major age groups, sex, and underlying comorbidities because patients who recently developed gout were enrolled. Furthermore, to increase the reliability of a diagnosis of PD, we extracted patients with incidental PD enrolled in the RID system. Additionally, by performing a subgroup analysis, we were able to elucidate other risk factors and protective factors for PD.

\section{Conclusions}

This population-based study did not identify an association between gout and PD. Clinical characteristics, including frequency according to age group and sex distribution in domestic patients with gout, were confirmed. Further, our analysis identified the percentage of cardiovascular and metabolic comorbidities. Age, female sex, stroke, and hypertension were identified as independent risk factors for PD, and dyslipidemia exhibited an inverse relationship with PD. Further research is warranted to verify our findings.

Supplementary Materials: The following are available online at https: / / www.mdpi.com/article/ 10.3390/medicina57121292/s1, Table S1: Cox proportional hazard model of risk factors for Parkinson's disease by sex.

Author Contributions: Conceptualization, J.H.K. and G.K.; methodology, J.H.K. and G.K.; formal analysis, J.H.K. and G.K.; resources, G.K.; data curation, J.H.K. and G.K.; writing—original draft preparation, J.H.K. and A.K.; writing-review and editing, J.H.K., I.A.C. and A.K.; visualization, J.H.K. and G.K.; supervision, I.A.C. and G.K.; project administration, J.H.K. and G.K. Translation, J.H.K. All authors have read and agreed to the published version of the manuscript.

Funding: This research received no external funding. 
Institutional Review Board Statement: This study was approved by the institutional review board of Chungbuk National University Hospital (CBNUH IRB No. 2020-08-007, https: / www.e-irb.com: 3443/devlpg/nlpgS100.jsp). Patient consent was not required as the study was based on secondary data released for research purposes.

Informed Consent Statement: Not applicable.

Data Availability Statement: Data cannot be shared publicly because they belong to the National Health Insurance Service (NHIS). There are ethical restrictions on sharing such a data set because these data contain potentially identifying or sensitive patient information. To request data from NHIS, researchers have to apply during the recruitment period and submit a research proposal. Raw data was available to researchers upon reasonable academic request and with the permission of the Korean NHIS Institutional Data Access (https:/ / nhiss.nhis.or.kr/bd/af/bdafa001lv.do). The authors had no special access privileges.

Conflicts of Interest: The authors declare no conflict of interest.

\section{References}

1. Kalia, L.V.; Lang, L.E. Parkinson's disease in 2015; evolving basic, pathological and clinical concepts in PD. Nat. Rev. Neurol. 2016, 12, 65-66. [CrossRef]

2. Berardelli, A.; Wenning, G.K.; Antonini, A.; Berg, D.; Bolem, B.R.; Bonfifati, V.; Brooks, D.; Burn, D.J.; Colosimo, C.; Fanciulli, A.; et al. EFNS/MDS-ES/ENS recommendations for the diagnosis of Parkinson's disease. Eur. J. Neurol. 2013, 20, 16-34. [CrossRef] [PubMed]

3. De Lau, L.M.; Breteler, M.M. Epidemiology of Parkinson's disease. Lancet Neurol. 2006, 5, 525-535. [CrossRef]

4. Zhang, Z.X.; Roman, G.C. Worldwide occurrence of Parkinson's disease: An updated review. Neuroepidemiology 1993, 12, 195-208. [CrossRef] [PubMed]

5. Preingsheim, T.; Jettet, N.; Frolkis, A.; Steeves, T.D. The prevalence of Parkinson's disease; a systematic review and meta-analysis. Mov. Disord. 2014, 29, 1583-1590. [CrossRef]

6. Han, S.L.; Kim, S.; Kim, H.T.; Shin, H.W.; Na, K.S.; Suh, H.S. Prevalence and incidence of Parkinson's disease and drug-induced parkinsonism in Korea. BMC Public Health 2019, 19, 1328. [CrossRef]

7. Roddy, E.; Choi, H.K. Epidemiology of gout. Rheum. Dis. Clin. N. Am. 2014, 40, 155-175. [CrossRef]

8. Maxwell, S.R.; Thomason, H.; Sandler, D.; Leguen, C.; Baxter, M.A.; Thorpe, G.H.G.; Jones, A.F.; Barnett, A.G. Antioxidant status in patients with uncomplicated insulin-dependent and non-insulin-dependent diabetes mellitus. Eur. J. Clin. Investig. 1997, 27, 484-490. [CrossRef]

9. Shen, L.; Ji, H.F.; Hf, J. Low uric acid levels in patients with Parkinson's disease: Evidence from meta-analysis. BMJ Open 2013, 3, e003620. [CrossRef]

10. Abraham, A.; Drory, V.E. Influence of serum uric acid levels on prognosis and survival in amyotrophic lateral sclerosis: A meta-analysis. J. Neurol. 2014, 261, 1133-1138. [CrossRef]

11. Schwarzschild, M.A.; Schiwid, S.R.; Marek, K.; Watts, A.; Lang, A.E.; Oakes, D.; Shoulson, I.; Ascherio, A.; Parkinson Study Group PRECEPT Investigators. Serum urate as a predictor of clinical and radiographic progression in Parkinson disease. Arch. Neurol. 2008, 65, 716-723. [CrossRef]

12. Jenner, P. Oxidative stress as a cause of Parkinson's disease. Acta Neurol. Scand. Suppl. 1991, 136, 6-15. [CrossRef]

13. Hwang, O. Role of oxidative stress in Parkinson's disease. Exp. Neurobiol. 2013, 22, 11-17. [CrossRef] [PubMed]

14. Jenner, P. Oxidative stress in Parkinson's disease. Ann. Neurol. 2003, 53, S26-S36. [CrossRef]

15. National Health Insurance Service (NHIS). History of the NHIS. Available online: http://www.nhis.or.kr/static/html/wbd/g/ a/wbdga0203.html (accessed on 11 April 2016).

16. Seong, S.C.; Kim, Y.Y.; Khang, Y.H.; Park, J.H.; Kang, H.J.; Lee, H.Y.; Do, C.H.; Song, J.S.; Hyon Bang, J.; Ha, S.; et al. Data Resource Profile: The National Health Information Database of the National Health Insurance Service in South Korea. Int. J. Epidemiol. 2017, 46, 799-800.

17. Singh, J.A.; Cleveland, J.D. Gout and the risk of Parkinson's disease in older adults: A study of U.S. Medicare data. BMC Neurol. 2019, 19, 4. [CrossRef] [PubMed]

18. Hu, L.Y.; Yang, A.C.; Lee, S.C.; You, Z.H.; Tsai, S.J.; Hu, C.K.; Shen, C.C. Risk of Parkinson's disease following gout: A population-based retrospective cohort study in Taiwan. BMC Neurol. 2020, 20, 338. [CrossRef]

19. Janssens, H.J.; Arts, P.G.; Schalk, B.W.; Biermans, M.C. Gout and rheumatoid arthritis, both to keep in mind in cardiovascular risk management: A primary care retrospective cohort study. Joint Bone Spine 2017, 84, 59-64. [CrossRef]

20. Sanchez-Lozada, L.G.; Rodriguez-Iturbe, B.; Kelley, E.E.; Nakagawa, T.; Madero, M.; Feig, D.I.; Borghi, C.; Piani, F.; Cara-Fuentes, G.; Bjornstad, P.; et al. Uric Acid and Hypertension: An Update with Recommendations. Am. J. Hypertens. 2020, 33, 583-594. [CrossRef]

21. Kutzing, M.K.; Firestein, B.L. Altered uric acid levels and disease states. J. Pharmacol. Exp. Ther. 2008, 324, 1-7. [CrossRef] [PubMed] 
22. Piani, F.; Cicero, A.F.G.; Borghi, C. Uric Acid and Hypertension: Prognostic Role and Guide for Treatment. J. Clin. Med. 2021, 10, 448. [CrossRef] [PubMed]

23. Harada, H.; Nishikawa, S.; Takahashi, K. Epidemiology of Parkinson's disease in a Japanese city. Arch. Neurol. 1983, 40, 151-154. [CrossRef] [PubMed]

24. Liu, C.C.; Li, C.Y.; Lee, P.C.; Sun, Y. Variations in incidence and prevalence of Parkinson's disease in Taiwan: A population-based nationwide study. Parkinson's Dis. 2016, 2016, 8756359. [CrossRef] [PubMed]

25. Wang, Y.; Shi, Y.; Wu, Z.; He, Y.; Zhang, B. Parkinson's disease in China. Chin. Med. J. 1991, 104, $960-964$.

26. Benito-Leon, J.; Bermejo-Pareja, F.; Morales-Gonzalez, J.; Porta-Etessam, J.; Trincado, R.; Vega, S.; Louis, E.D. Incidence of Parkinson disease and parkinsonism in three elderly populations of Central Spain. Neurology 2004, 62, 734-741. [CrossRef]

27. Nerius, M.; Fink, A.; Doblhammer, G. Parkinson's disease in Germany: Prevalence and incidence based on health claims data. Acta Neurol. Scand. 2017, 136, 386-392. [CrossRef]

28. Marras, C.; Beck, J.C.; Bower, J.H.; Roberts, E.; Ritz, B.; Ross, G.W.; Abbott, R.D.; Savica, R.; Van Den Eeden, S.K.; Willis, A.W.; et al. Prevalence of Parkinson's disease across North America. NPJ Parkinson's Dis. 2018, 4, 21. [CrossRef]

29. Bauso, D.; Tartari, J.; Stefani, C.; Rojas, J.; Giunta, D.; Cristiano, E. Incidence and prevalence of Parkinson's disease in Buenos Aires City. Argent. Eur. J. Neurol. 2012, 19, 1108-1113. [CrossRef]

30. Hu, G.; Antikainen, R.; Jousilahti, P.; Kivipelto, M.; Tuomilehto, J. Total cholesterol and the risk of Parkinson disease. Neurology 2008, 70, 1972-1979. [CrossRef]

31. Guo, X.; Song, W.; Chen, K.; Chen, X.; Zheng, Z.; Cao, B.; Huang, R.; Zhao, B.; Wu, Y.; Shang, H.-F. The serum lipid profile of Parkinson's disease patients: A study from China. Int. J. Neurosci. 2015, 125, 838-844. [CrossRef]

32. Rozani, V.; Gurevich, T.; Giladi, N.; El-Ad, B.; Tsamir, J.; Hemo, B.; Peretz, C. Higher serum cholesterol and decreased Parkinson's disease risk: A statin-free cohort study. Mov. Disord. 2018, 33, 1298-1305. [CrossRef] [PubMed]

33. Fang, F.; Zhan, Y.; Hammar, N.; Shen, X.; Wirdefeldt, K.; Walldius, G.; Mariosa, D. Lipids, apolipoproteins, and the risk of Parkinson disease. Circ. Res. 2019, 125, 643-652. [CrossRef] [PubMed]

34. Huang, X.; Auinger, P.; Eberly, S.; Oakes, D.; Schwarzschild, M.; Ascherio, A.; Mailman, R.; Chen, H.; Parkinson Study Group DATATOP Investigators. Serum Cholesterol and the Progression of Parkinson's Disease: Results from DATATOP. PLoS ONE 2011, 6, e22854. [CrossRef] [PubMed]

35. Barres, B.A.; Smith, S.J. Cholesterol-Making or breaking the synapse. Science 2001, 294, 12961297. [CrossRef] [PubMed]

36. Thiele, C.; Hannah, M.J.; Fahrenholz, F.; Huttner, W.B. Cholesterol binds to synaptophysin and is required for biogenesis of synaptic vesicles. Nat. Cell Biol. 2000, 2, 4249. [CrossRef]

37. Stoffel-Wagner, B. Neurosteroid metabolism in the human brain. Eur. J. Endocrinol. 2001, 145, 669679. [CrossRef]

38. Rouge-Pont, F.; Mayo, W.; Marinelli, M.; Gingras, M.; Le Moal, M.; Piazza, P.V. The neurosteroid allopregnanolone increases dopamine release and dopaminergic response to morphine in the rat nucleus accumbens. Eur. J. Neurosci. 2002, $16,169173$. [CrossRef]

39. Barrot, M.; Vallee, M.; Gingras, M.A.; Le Moal, M.; Mayo, W.; Piazza, P.V. The neurosteroid pregnenolone sulphate increases dopamine release and the dopaminergic response to morphine in the rat nucleus accumbens. Eur. J. Neurosci. 1999, 11, 37573760. [CrossRef]

40. Shalaby, S.Y.; Louis, E.D. Statin use and its association with essential tremor and Parkinson's disease. Neuroepidemiology 2016, 47, 11-17. [CrossRef]

41. Seng, Z.; Jia, X.; Kang, M. Statin use and risk of Parkinson's disease: A meta-analysis. Behav. Brain Res. 2016, 309, 29-34. [CrossRef]

42. Belvisi, D.; Pellicciari, R.; Fabbrini, G.; Tinazzi, M.; Berardelli, A.; Defazio, G. Modifiable risk and protective factors in disease development, progression and clinical subtypes of Parkinson's disease: What do prospective studies suggest. Neurobiol. Dis. 2020, 134, 104671. [CrossRef] [PubMed]

43. Davies, K.J.; Sevanian, A.; Muakkassah-Kelly, S.F.; Hochstein, P. Uric acid-iron ion complexes. A new aspect of the antioxidant functions of uric acid. Biochem. J. 1986, 235, 747-754. [CrossRef] [PubMed]

44. Waring, W.S. Uric acid: An important antioxidant in acute ischemic stroke. QJM 2002, 95, 691-693. [CrossRef] [PubMed]

45. Wang, N.; Jin, X.; Guo, D.; Tong, G.; Zhu, X. Iron chelation nanoparticles with delayed saturation as an effective therapy for Parkinson Disease. Biomacromolecules 2017, 18, 461-474. [CrossRef] [PubMed]

46. Dusek, P.; Schneider, S.A.; Aaseth, J. Iron chelation in the treatment of neurodegenerative diseases. J. Trace Elem. Med. Biol. 2016, 38, 81-92. [CrossRef] 